\title{
Editorial
}

\section{Mindfulness, bienestar y salud}

\author{
Dr. Manuel Rodríguez Zapata ${ }^{1}$ y Dr. Juan-Carlos Luis-Pascual2,* \\ 1 Decano de la Facultad de Medicina y Ciencias de la Salud, Universidad de Alcalá. \\ 2 Vicedecano de la Facultad de Medicina y Ciencias de la Salud, Universidad de Alcalá. \\ * Autor correspondencia: juan.luis@uah.es
}

La práctica formal comienza a ser una constante en diferentes ámbitos de la vida: en la empresa, en la educación, en el deporte y también en la salud. Es excesivamente simplista reducir el concepto de mindfulnes al entrenamiento de la atención plena, aunque sólo fuera esto y de una manera pragmática hay una gran cantidad de evidencia empírica del impacto que supone para la mejora de la salud psicosomática tanto en población clínica como no clínica.

Una primera aproximación conceptual lleva a orientar nuestra atención de manera voluntaria a lo que hacemos en cada momento sin llevar a cabo enjuiciamientos, pero en una segunda revisión tratando de profundizar un poco más, nos encontramos con que podría ser un complemento multimodal a la terapia farmacológica utilizada y empleada por el personal sanitario con sus pacientes. No obstante, esta práctica podría llegar a tener efectos terapéuticos para los participantes no clínicos que buscan aligerar el peso de sus "rumiaciones" sobre lo que le ha ocurrido en el pasado o la ansiedad provocada por lo que pueda suceder en el futuro.

Quizás solo interesa abandonar en algunos momentos el modo hacer, que nos lleva a vivir de una manera automática y no consciente, permitiéndonos ocasionalmente, al menos durante la práctica formal estar en modo ser, percibiendo más las sensaciones físicas, los sonidos, las emociones, o el reflejo de éstas en nuestro cuerpo, observando desde fuera nuestros pensamientos, desidentificándonos de ellos. La propuesta esencial es tener una actitud abierta y no reactiva que experimente sin pre-juicios y dejando a un lado las experiencias pasadas que nos permita observar plenamente la frescura de cada momento.

Lo cercano y lo cotidiano, que ahora no somos capaces de "ver" emergerá ante nuestros ojos con interés y relevancia. Tan sólo tenemos que generar un pequeño espacio a la consciencia que evite la reacción y la toma de control de los pensamientos y emociones que tenemos sobre nuestras respuestas, tomando cierta distancia objetiva de ellos.

En la Universidad de Alcalá se ha iniciado una revolución silenciosa que pretende implementar el mindfulness, a través de un proyecto de innovación, como mejora del clima de aprendizaje en el aula y que facilite incrementar el bienestar del profesorado. La sistematización de una práctica cotidiana formal o informal de respiración, relajación y atención plena del personal de la Universidad podría conseguir hacernos más competentes para prevenir el estrés y la ansiedad.

Este monográfico de mindfulness y compasión es un ejemplo claro de la difusión y dinamización que se está llevando a cabo. Entre todas estas actividades formativas y grupos de práctica destacan la II Jornada de Mindfulness, bienestar y salud [1], los Talleres de mindfulness para los PAS y los PDI [2], de una hora semanal, organizados por el CAD y realizados en la Facultad de Medicina y Ciencias de la Salud y en la Facultad de Educación, la incorporación de la asignatura transversal Mindfulness, bienestar y salud [3] ofertada para los estudiantes de la Universidad, el Taller de Mindfulness y Escuela Relajada en el Programa de la Universidad de los niños, el curso de ocho semanas certificado por el ICE de la Universidad de Alcala: MBI-Mindfulness: formación básica en atención plena para maestros [4], para los profesores tutores que colaboran en las prácticas docentes de los diferentes grados de Magisterio, en el Módulo III y IV del Máster Universitario de Acción Humanitaria Sanitaria [5], en la asignatura optativa Técnicas de relajación aplicadas a la Educación física, del Máster Universitario de formación del profesorado [6], o en el Máster de Mindfulness y Compasión [7]. 
Elaborando materiales para facilitar la práctica formal del mindfulness, a través de audios para smartphone como Ivoox [8] o vídeos en Youtube [9].

Entre los antecedentes académicos de mindfulness en otras universidades extranjeras podemos destacar: Universidad de Bangor (Gran Bretaña) [10], Universidad de California (Estados Unidos) [11], Universidad de Exeter (Gran Bretaña) [12], Universidad de Harvard (Estados Unidos) [13], Universidad de Oxford (Gran Bretaña) [14]. Y entre los antecedentes académicos del mindfulness en universidades españolas tenemos, por orden alfabético: Universidad de Alcalá [15], Universidad de Barcelona [16], Universidad Complutense [17], Universidad de Valencia [18] o la Universidad de Zaragoza [19].

\section{Enlaces, materiales y recursos}

1. García-Campayo J (2019). Conferencia de la evidencia empírica de los efectos del mindfulness en la salud. http://portalcomunicacion.uah.es/diario-digital/actualidad/conferencia-sobremindfulness-y-salud-en-el-rectorado-de-la-uah.html

2. Talleres de mindfulness para PAS y PDI http://www3.uah.es/cadccs/inscripciones/taller-demindfulness-2018-2019/

3. Asignatura Transversal Mindfulness, bienestar y salud https://www.uah.es/export/sites/uah/es/estudios/estudiosoficiales/grados/galleries/Programas/G61/100175_G61_2018-19.pdf

4. Curso para profesores no universitarios MBI-Mindfulness: Formación básica en atención plena para maestros

https://www3.uah.es/ice/FP/documentos/MBI_MINDFULNESS_FORMACI\%C3\%93N_B\%C3\%81SICA_A TENCI\%C3\%93N_PLENA_MAESTROS.pdf

5. Módulo III Máster Universitario de Acción Humanitaria Sanitaria

https://www.uah.es/es/estudios/Accion-Humanitaria-Sanitaria/

6. Asignatura Optativa Técnicas de relajación aplicadas a la educación física

http://www3.uah.es/master_fps/documentos_pdf/programas/educacion_fisica/tecnicas_relajacion.pdf

7. Máster de Mindfulness y Compasión https://www.uah.es/es/estudios/estudios-propios/posgradospropios/Master-en-Mindfulness-y-Compasion/

8. Ivoox Mindfulness y Escuela Relajada

https://www.ivoox.com/podcast-escuela-relajada_sq_f1581698_1.html

9. YouTube Mindfulness y Escuela Relajada

https://www.youtube.com/channel/UCEk1ngbVKQ_Qw3Q6di5yWRQ

10. Universidad de Bangor (Gran Bretaña) https://www.bangor.ac.uk/mindfulness/

11. Universidad de California (Estados Unidos) http://marc.ucla.edu/mindful-meditations

12. Universidad de Exeter (Gran Bretaña) http://cedar.exeter.ac.uk/mindfulness/

13. Universidad de Harvard (Estados Unidos) https://www.health.harvard.edu/blog/mindfulnessmeditation-may-ease-anxiety-mental-stress-201401086967

14. Universidad de Oxford (Gran Bretaña) http://www.ox.ac.uk/news/2015-07-16-large-scale-trial-willassess-effectiveness-teaching-mindfulness-uk-schools

15. Universidad de Alcalá https://www.uah.es/es/estudios/estudios-propios/posgrados-propios/Masteren-Mindfulness-y-Compasion/

16. Universidad de Barcelona

http://www.ub.edu/web/ub/es/estudis/oferta_formativa/masters_propis/fitxa/R/201711672/index.html

17. Universidad Complutense https://www.ucm.es/cseg/experto-en-mindfulness-en-contextos-de-salud

18. Universidad de Valencia https://postgrado.adeituv.es/es/cursos/psicologia-5/mindfulness/datos_generales.htm

19. Universidad de Zaragoza http://www.masterenmindfulness.com/ 
(C) 2019 por los autores; Esta obra está sujeta a la licencia de Reconocimiento 4.0 Internacional de Creative Commons. Para ver una copia de esta licencia, visite http://creativecommons.org/licenses/by-nc-nd/4.0/. 